\title{
The ACS LCID project: data reduction strategy
}

\author{
M. Monelli ${ }^{1}$ for the LCID team $\dagger$ \\ ${ }^{1}$ Insituto de Astrofísica de Canarias, C/Via Lactea s/n, 38205 La Laguna, Tenerife, Spain \\ email: monelli@iac.es
}

\begin{abstract}
During Cycle 14 a total of 113 HST orbits were secured to observe five isolated dwarf galaxies, namely Tucana, LGS3, LeoA, IC1613, and Cetus. The aim of the project is a full characterization of the stellar content of these galaxies, in term of their SFH, radial distributions, halo populations and variable stars. Deep (V $\approx 29)$ F475W, F814W data allowed us to fully sample all the evolutionary phases from the tip of the Red Giant Branch (RGB) to well below the old Main Sequence Turnoff (MSTO). Here we describe the observational design, and the reduction and calibration strategy adopted. A comparison of the results obtained using two different packages, ALLFRAME and Dolphot, is presented.
\end{abstract}

Keywords. (galaxies:) Local Group, galaxies: photometry, techniques: photometric, galaxies: individual (Tucana)

The main goal of this project (Gallart et al., this proceedings) is to obtain deep colormagnitude diagrams (CMDs) reaching the oldest MSTO $\left(\mathrm{M}_{I} \simeq+3\right)$, in order to obtain detailed SFHs and to perform comparative studies within the sample and with available data for Milky Way satellites. The SFH will be recovered from the global analysis of the star distribution on the CMD, using IAC-star and IAC-pop (Aparicio et al., this proceedings). The observational design was determined as follows. The most challenging part of our project was to trace age differences at old ages, and we found that the largest age sensitivity of old isochrones occurs as color differences at $M_{I} \simeq+2.75$. We also determined that the F475W and F814W filters is the most efficient combination to trace it. We calculated that, in order to trace age differences of about 2 Gyr at the above magnitude level, a precision of $\simeq 0.1$ magnitudes was necessary. This determined the number of orbits devoted to each object. Moreover, these exposure times allow us to reach $\mathrm{M}_{I} \simeq+2.5$ with $\mathrm{S} / \mathrm{N} \simeq 10$ in the parallel WFPC2 exposures, using the $\mathrm{F} 450 \mathrm{~W}$ and $\mathrm{F} 814 \mathrm{~W}$ filters. The last are expected to produce CMDs showing stars down to $\simeq 5$ Gyr on the MS, but with the old MSTOs below the limiting magnitude. The WFPC2 parallels were strategically placed in order to sample the outer parts of each galaxy, and thus to provide information on stellar population gradients. In the case of the smallest objects, they may provide limits to the extent of the galaxy.

The photometry of these data is being performed in parallel using two different software packages, namely DAOPHOT/ALLFRAME (Stetson 1994) and Dolphot, an adaptation of HSTphot (Dolphin 2000) to the perform ACS photometry. This will allow us to assess the possible systematic effects on the CMD, which may affect the SFH derivation. We adopted similar approaches with both packages. After many tests, we decided to work with the original _FLT images rather the drizzled ones. The PSF was modeled individually for each image, and the input list of stars was obtained from the cosmicray-cleaned stacked median image. The photometric calibration was performed in the VEGAMAG system, following the prescriptions given by Sirianni et al. (2005). Fig. 1

$\dagger$ Local Cosmology from Isolated Dwarfs, http://www.iac.es/project/LCID 

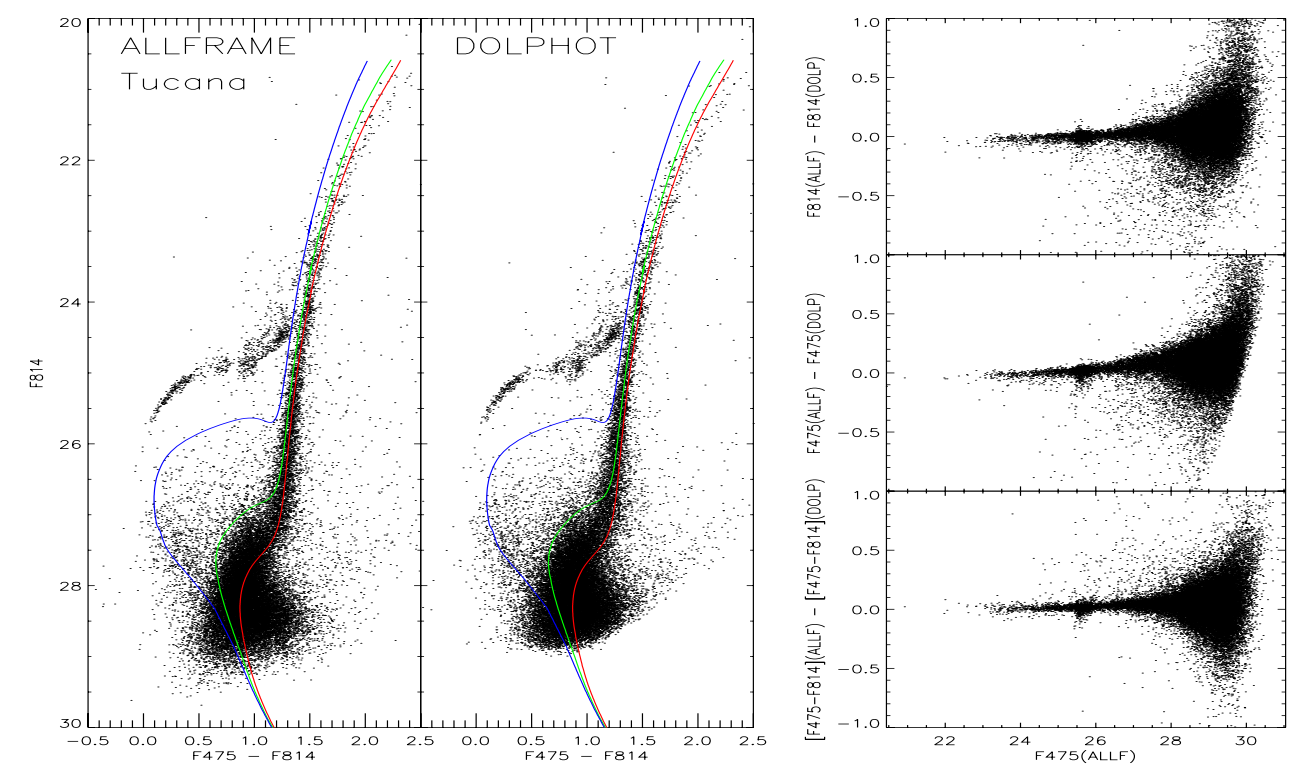

Figure 1. Left - Allframe and Dolphot CMDs. The same theoretical isocrones $(\mathrm{Z}=0.006, \mathrm{t}=11$, 7, 3.5 Gyr, $\left.\mathrm{DM}_{0}=24.7, \mathrm{E}(\mathrm{B}-\mathrm{V})=0.03\right)$ from the BASTI database have been superimposed on both plots. Right - Magnitude and color differences as a funcion of $F 475 \mathrm{~W}$ magnitude.

(left) shows the comparison between the Tucana CMD obtained with the two methods. The right panels show, as a function of the $F 475 W$ magnitude, the $\Delta(F 475 W)$, $\Delta(F 814)$, and $\Delta(F 475 W-F 814 W)$. The comparison discloses a possible small zero point and a systematic trend towards faint magnitudes, being ALLFRAME magnitudes fainter then Dolphot ones. However, this trend appears to be similar in both bands, and appears not to significantly affect the color. The differences between the results of the two packages are small, and this should not affect the derived SFH in any way. To qualitatively show this, theoretical isochrones from the BASTI database (Pietrinferni et al. 2004, http://www.te.astro.it/BASTI/index.php) are superimposed on the CMDs. Extensive experiments are being performing to set this point on a quantative basis.

\section{Acknowledgements}

This work was partially supported by spanish MEC (AYA2004-06343) the IAC (3I0394), by the European Structural Funds and NASA through grants GO-10505 and GO-10590

\section{References}

Dolphin, A.E. 2000, PASP, 112, 1383

Pietrinferni, A., Cassisi, S., Salaris, M., \& Castelli, F. 2004, ApJ, 612, 168

Sirianni, M., Jee, M. J., Bentez, N.,Blakeslee, J. P., Martel, A. R., Meurer, G., Clampin, M., De Marchi, G., Ford, H. C., Gilliland, R., Hartig, G. F., Illingworth, G. D., Mack, J., \& McCann, W. J. 2005, PASP, 117, 1049

Stetson, P.B. 1994, PASP, 106, 250 